\title{
The Study on the Piano Sound Control and the Computer Simulation
}

\author{
Qihe Zhang ${ }^{1, \text { a }}$ \\ ${ }^{1}$ The College of Continuing Education Binzhou Polytechnic Shandong Binzhou China 256603 \\ a619511537@qq.com
}

Keywords: The piano; Voice control; Computer; Simulation

\begin{abstract}
The art is the heart of desire, the pursuit of truth, kindness and beauty, and the love of life. With the gradual improvement of the material life, people, especially in the field of mental pursuit of beauty are becoming higher. As the king of musical instruments piano was first popular and accepted, the beauty of the music of the piano with its unique color and tone. Pseudo acoustic modeling can only to a certain extent, acoustic parameters of instrument simulation, under the condition of reality there are many factors which can affect the instrument vibration and sound, virtual modeling technology and is not to be completely covered. This article will discuss the piano sound control and computer simulation.
\end{abstract}

\section{Introduction}

Along with the increasing abundance of material civilization, people pursuit of spiritual civilization is becoming much higher. And the piano for its wide range sounds beautiful melody, good reputation, has been dubbed the "king of Musical Instruments into more and more families. However, the keys of the piano tone phrase as a failure of the beautiful picture, intolerable. The strings of a piano has the ductility of the metal, will happen under the great tension for a long time to extend and deformation cause pitch, it is an important cause of the change of the piano intonation. So no matter whether to play the piano, after a period of time should be carried out on the piano tuning. For piano beginners, they form a good enlightenment stage intonation concepts and important stages of music feeling. If user tone-deaf piano to training did not develop the music hearing, hearing over time will be not standard music wrongly guide. 's sense of the concept of pitch error, especially intonation concept aspect, this will cause problems for future study and regret. Therefore, for beginners of piano, best in the six months to one year's time, adjust the piano at a time.

\section{The Overview of the Piano Tuning System}

Piano tuning system is through the computer to realize a key in a piano voice signal real-time analysis, calculate the single tone pitch frequency, and the standard of pitch frequency contrast, show the error as a reference the tuning, and correspondingly adjust the tightness of the strings, and then repeating the process, until the sound and tune in theory allows within the scope of the standard error, the end of the tuning keys. Including the system's overall function:

Parameter Settings: this step is to pitch below segment detection, particular way is a piano in the aspect of software design model, the response function of each key Settings have pitch standard frequency, detection range, and some parameters such as piano sound. In each corresponding to the press before tuning a piano key, standard pitch frequency and range of these parameters can be attached to the initial value, these parameters are set as a global variable, so that in can also directly led to the subsequent processing.

Signal acquisition: using ordinary microphone to complete the voice signal into electrical signal conversion, the microphone is the input device of this system. Analog-to-digital conversion process is done through the sound card, set various parameters during the process of collecting can be control by with Matlab toolbox function. 
Signal preprocessing: in order to reduce the piano pitch detection signal noise interference, when the need to advance the piano signal filtering, filter out higher harmonic and noise. Also, to make frame the entire length of the signal processing, divided into a frame of a frame of short-term stationary signal.

Endpoint detection: the purpose is to find the starting position of the piano sound signal, thus the useful signal extracted from the entire length of the signal. Specific means is short-time energy and short-time zero crossing ratio test.

Pitch detection: to extract the useful signal, make sure the sound is low frequency or high frequencies, and then according to the different frequency band, low frequency band with the improved autocorrelation method, the high frequency harmonic peak method, and the pitch frequency.

Results show that the interface through Matlab shows that the measured frequency values, the relative error, and the time and frequency domain signal waveform figure.

\section{Analysis on the Virtual Modeling Process of the Soundboard}

In order to verify the virtual modeling method in the role of the piano soundboard vibration modal analysis, the experiment method is for the actual testing. With a domestic manufacturer production upright piano 123 model as an example, the finite element analysis software ANSYS virtual modeling analysis was carried out on the soundboard.

(1) Defining the research object and the object of study on the measured some brand upright piano soundboard back.

(2) Drawing 2D CAD floor plan.

(3) $3 \mathrm{~d}$ modeling and import the finite element analysis software ANSYS model is established in this paper.

(4) Defining the soundboard materials is the most commonly used material physical properties of the piano soundboard spruce.

(5) Meshing and applied load was carried out on the soundboard after the automatic meshing applied load, as shown in figure 1.

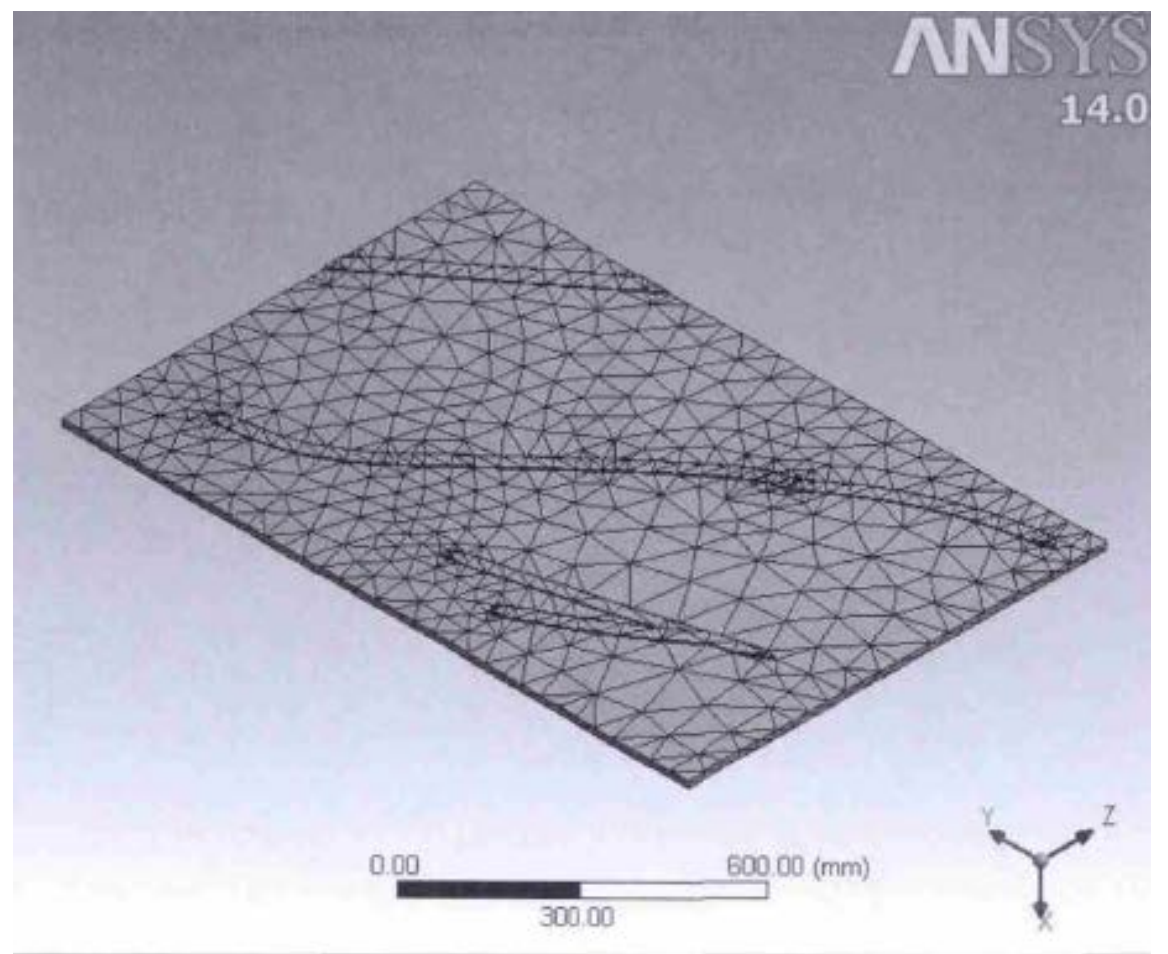

Figure 1 ANSYS model

The piano in the process of production and processing, soundboard quadrilateral to directly glued the soundboard box; this is the soundboard boundary conditions. Because the strings hanging 
in iron can be higher than pillow and hanging strings are nails and produce Angle, so the pressure to string code generation, and conduction to the soundboard. Many studies of soundboard often ignore the pressure; it had a great influence on the piano timbre. Many writings discussed pressure, means the string through the string codes for the pressure of the soundboard. Can through the static of the vertical pressure is calculated. In the process of load application, you must pay attention to the role of the parameters in the modal analysis. Through calculation, from higher code to bass string yards evenly applied force $29.4 \mathrm{~N} \sim 58.8 \mathrm{~N}$.

(6) Solving. After putting constraints, is solved, extension mode for 6 order, soundboard is obtained in the first six order natural frequency. The first order resonance frequency is $72.221 \mathrm{~Hz}$, for the whole soundboard deformation deflection of great value, there is only one place it is located in the center of the whole piece of soundboard; Second order resonance frequency of $111.49 \mathrm{~Hz}$, second order vibration mode in this picture you can see in the middle of the soundboard form a line vibration node, a transverse vibration mode; The third order resonance frequency is $183.27 \mathrm{~Hz}$, the third order modes can be seen that is transverse vibration mode, by the two nodes will soundboard is divided into three vibration area; The fourth order resonance frequency is $189.21 \mathrm{~Hz}$, fourth order resonance for longitudinal vibration mode, is a section of line is divided into two Fang Zhen area; The fifth order modal vibration frequency of $229.8 \mathrm{~Hz}$, by two section lines are divided into four vibration area; Sixth order modal frequency is $293.75 \mathrm{~Hz}$, is three line section is divided into four vibration area, the transverse vibration model. Figure 2 is the frequency diagram of the piano keys

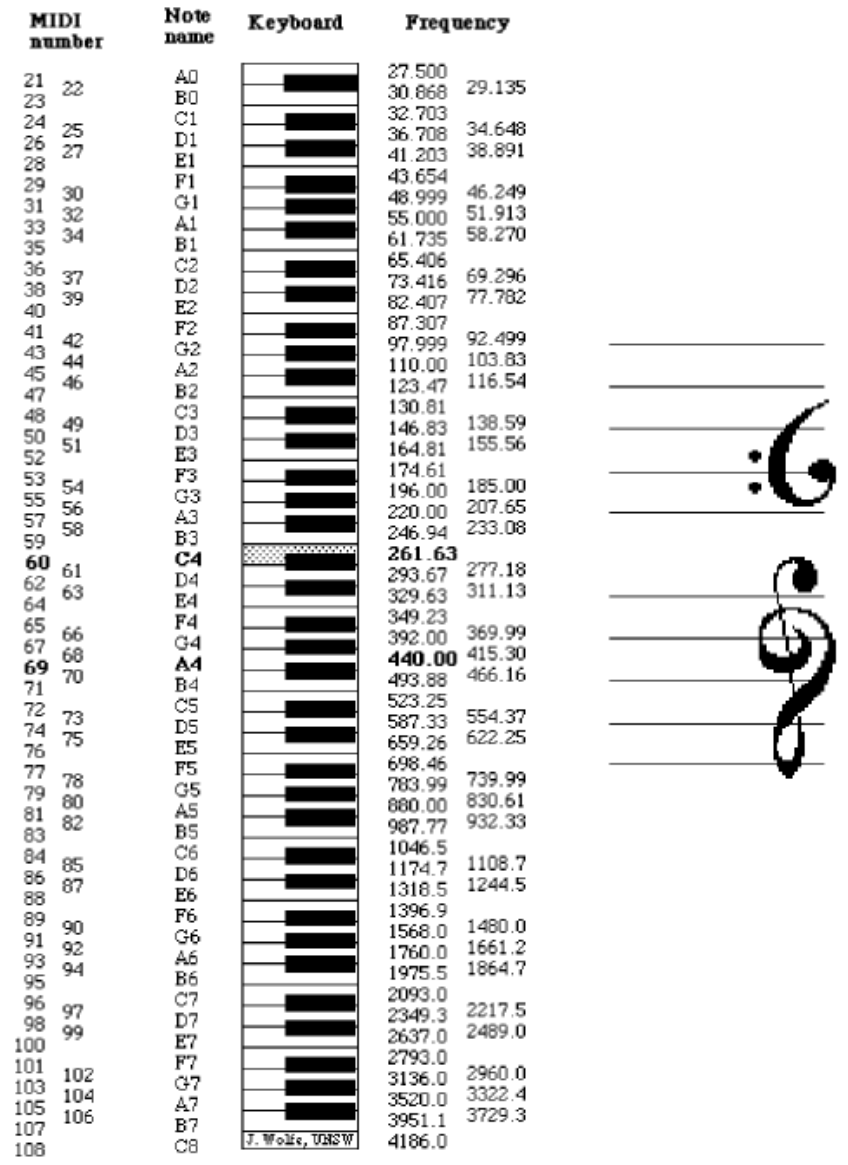

Figure 2The frequency diagram of the piano keys

\section{The Sound Acquisition System based on the Sound Card and Matlab}

Voice signal acquisition and analysis in engineering application is often need to solve the problem, how to collect real-time voice signal processing and analysis, find out the characteristics of the sound signal in scientific research is a very meaningful work. Matlab Toolbox provides a Data collection, Data Acquisition Toolbox), in the Toolbox, the Data collection is a set of commands and functions, can be used to directly control compatible with PC Data Acquisition 
equipment for Data Acquisition, therefore, using a Toolbox of Matlab voice signal Acquisition. Then called directly in Matlab functions, power spectrum analysis, spectrum analysis and numerical analysis function and so on, can be separately collected voice signal spectrum, power spectrum analysis and so on the many kinds of spectrum analysis. Therefore, it can be easily implemented in the Matlab signal acquisition and analysis work. Can send control commands to the sound card in the process of acquisition, such as suspension sampling, exit, etc. Data collected were temporarily stored in computer memory and can theoretically the maximum number of data collection is determined by the computer's memory. Hardware attributes of the device at the same time, Matlab can record collection and gathering the startup time, collecting the information such as time, sampling frequency and sampling channel, if the wrong appeared in the process of acquisition, the wrong time, wrong information such as the source also can be recorded for subsequent work reference. It is important to note that performed a data acquisition device object should be deleted after work, will be stored on a hard disk data in memory after release the data storage memory space occupied, in preparation for the next gathering enough memory space to store the new data.

Pitch detection is essentially to the music signal pitch detection. The so-called pitch also call tones, it is the human ear to distinguish a measurement of vibration frequency noise meter. Pitch is mainly depends on the high and low frequency. Compared with the voice signal, the periodic music signal is relatively stable, pitch of each sound is approximate fixed, its approximate spectrum is discrete spectrum, so it can detect the basic sound of music signal is more precise. For the piano after initial setting, the actual pitch frequency of each key will fall in the adjacent two keys of the standard pitch frequency range. If know the name of the key, just between the two neighbors standard frequency pitch to find the key. In other words, two adjacent keys standard frequency corresponding to the sampling values of the correlation function to find the key doesn't pitch, so we can in advance of each key $\mathrm{P}$ value is set a range $(\mathrm{n} 1$, and $\mathrm{n} 2)$, generally we select ideal $\mathrm{P}$ values before and after 50 a.m., to find the maximum value in the more than 100 points. This will greatly improve processing speed, and largely reduce the noise effects.

\section{Summary}

The piano sound and voice have many characteristics, such as they follow the general model of vibration pronunciation, dullness and piano sound components are very similar, are composed of fundamental wave and multiple harmonics. The piano tuning is the key to pitch frequency detection, and pitch detection in speech recognition is a key technology. It provides a theoretical basis for the research work. But the piano sound is than voice more broad frequency range, and the sampling frequency is limited, the estimate on the pitch was adopted when the segmented detection method, namely the low frequencies with the improved autocorrelation algorithm, peak method using high frequency harmonic. It is innovation of this article.

\section{References}

[1] Kim Weaver,Khurram Waheed,Fathi M Salem.An entropy based robust speech boundary detection algorithm for realistic noisy environments. ICSP 2012.

[2] Kashino Kunio,Murasc Hiroshi.Music recognition using note transition context. IEEE International Conference on Acoustics Speech and Signal Processing Proceedings. 2012

[3] Masoud Alghomemy,Ahmed H Tewfik.Rhythm and periodicity detection in polyphonic music IEEE signal Processing. Society 1999 Workshop on Multimedia Signal Processing. 2010

[4] Moorer.An Approach for the Separation of Voices in Composite Musical Signals. 2011

[5] Margaret L Johnson.Toward an expert system for expressive musical performance. IEEE Computer. 2012 